\title{
Predictions for high-frequency radio surveys of extragalactic sources
}

\author{
G. De Zotti ${ }^{1,2}$, R. Ricci ${ }^{2}$, D. Mesa ${ }^{1}$, L. Silva ${ }^{3}$, P. Mazzotta $^{4}$, L. Toffolatti ${ }^{5}$, and J. González-Nuevo ${ }^{5}$ \\ 1 INAF - Osservatorio Astronomico di Padova, Vicolo dell'Osservatorio 5, 35122 Padova, Italy \\ e-mail: dezotti@pd.astro.it \\ 2 International School for Advanced Studies, SISSA/ISAS, via Beirut 2-4, 34014 Trieste, Italy \\ e-mail: ricci@sissa.it \\ 3 INAF - Osservatorio Astronomico di Trieste, via G.B. Tiepolo 11, 34131 Trieste, Italy \\ e-mail: silva@ts.astro.it \\ ${ }^{4}$ Departimento di Fisica, Università di Roma "Tor Vergata", via della Ricerca Scientifica 1, 00133 Roma Italy \\ e-mail: pasquale.mazzotta@roma2.infn.it \\ 5 Departamento de Física, Universidad de Oviedo, c. Calvo Sotelo s/n, 33007 Oviedo, Spain
}

Received 2 October 2004 / Accepted 22 October 2004

\begin{abstract}
We present detailed predictions of the contributions of the various source populations to the counts at frequencies of tens of GHz. New evolutionary models are worked out for flat-spectrum radio quasars, BL Lac objects, and steepspectrum sources. Source populations characterized by spectra peaking at high radio frequencies, such as extreme GPS sources, ADAF/ADIOS sources and early phases of $\gamma$-ray burst afterglows are also dealt with. The counts of different populations of star-forming galaxies (normal spirals, starbursts, high- $z$ galaxies detected by SCUBA and MAMBO surveys, interpreted as proto-spheroidal galaxies) are estimated taking into account both synchrotron and free-free emission, and dust re-radiation. Our analysis is completed by updated counts of Sunyaev-Zeldovich effects in clusters of galaxies and by a preliminary estimate of galactic-scale Sunyaev-Zeldovich signals associated to proto-galactic plasma.
\end{abstract}

Key words. galaxies: active - galaxies: quasars: general - galaxies: BL Lacertae objects: general - radio continuum: general

\section{Introduction}

The new broad-band correlators for compact interferometric arrays and multi-beam receivers are beginning to enable large area surveys at high radio frequencies $(\geq 15 \mathrm{GHz})$, hitherto impossible because the beam solid angle scales as $v^{-2}$ (assuming diffraction limited performance) so that surveying large areas of the sky becomes quickly impractical at high frequencies with conventional techniques. The high-frequency surveys will have a major impact on astrophysics:

- They will open a window on new classes of both Galactic and extragalactic sources, for example on those with strong synchrotron or free-free self-absorption corresponding to both very early phases of nuclear radio-activity (extreme GHz Peaked Spectrum - GPS - sources or high-frequency peakers) and late phases of the evolution of Active Galactic Nuclei (AGNs), characterized by low accretion/radiative efficiency (ADAF/ADIOS sources), as well as to early phases of the evolution of radio afterglows of gamma-ray bursts.

- They will provide adequate samples to get a really unbiased view of rare, but very interesting, classes of sources with flat spectrum up to high frequencies, that, at low frequencies, are swamped by more numerous populations which fade away as the frequency increases. One example are blazars, for which much more extended samples are required to properly cover their parameter space. Galactic sources with substantial high-frequency emission include star-forming regions, transient black hole binaries and flaring brown dwarves.

- They will allow us to get important information even on the physics of sources known from lower frequency surveys: the high frequency spectral steepenings due to electron aging are informative on the distribution of source ages and on mechanisms for energy losses; the break frequencies marking the transition from optically thick to optically thin regimes depend on the magnetic field intensity, etc.

- They will produce surveys of the Sunyaev-Zeldovich effect in distant clusters of galaxies and perhaps on galactic scales, extremely important both to understand the formation of large scale structure and the heating of the intergalactic medium.

- They will play a vital role in the interpretation of temperature and polarization maps of the Cosmic Microwave Background (CMB), by allowing us to characterize and remove the contamination by astrophysical foregrounds. 
- They will be essential to calibrate the forthcoming large millimeter array ALMA.

- Follow-up high frequency observations will allow us to study the intrinsic polarization of sources, unaffected by Faraday depolarization, as well as their variability properties, essential ingredients to understand their physics.

NASA's WMAP satellite, designed to make accurate measurements of the space distribution of the Cosmic Microwave Background (CMB), is carrying out the first all sky millimeter surveys in five bands (centered at 22.8, 33.0, 40.7, 60.8, and 93.5 GHz; Bennett et al. 2003). The analysis of the first year observations has yielded a $98 \%$ reliable catalog of 208 point sources brighter than $\sim 1 \mathrm{Jy}$.

Unbiased radio surveys at $15 \mathrm{GHz}$, covering $520 \mathrm{deg}^{2}$, have been carried out with the Ryle telescope (Taylor et al. 2001; Waldram et al. 2003). Ricci et al. (2004), using a novel wide-band analogue correlator on one baseline of the Australia Telescope Compact Array (ATCA) have surveyed $1216 \mathrm{deg}^{2}$ of the southern sky at $18 \mathrm{GHz}$. Preliminary source counts at $30 \mathrm{GHz}$ have been produced by the DASI (Kovac et al. 2002), VSA (Grainge et al. 2003), and CBI (Mason et al. 2003) CMB experiments.

The ATCA survey will cover, in the near future, $10^{4} \mathrm{deg}^{2}$ at $20 \mathrm{GHz}$ to a flux limit $S_{20 \mathrm{GHz}}>40 \mathrm{mJy}$. The Jodrell Bank Observatory - University of Torun One Centimetre Radio Array (OCRA) will begin soon a multi-beam survey of the Northern sky at $30 \mathrm{GHz}$. ESA's PLANCK satellite will carry out all-sky surveys at 9 frequencies from 30 to $860 \mathrm{GHz}$ with sensitivity several times higher than WMAP.

As a new observational window opens up, it is useful to utilize models exploiting the available information to foretell which source populations the planned surveys will be able to detect as a function of their depth, which redshift range will these survey explore, which information on formation and evolution of each source population will they be able to provide. Answering these questions is a key step towards an optimal planning of the surveys.

Earlier time-honored comprehensive analyses of radiosource evolution (Dunlop \& Peacock 1990; Toffolatti et al. 1998; Jackson \& Wall 1999) provided remarkably successful fits to data from surveys at $\lesssim 8 \mathrm{GHz}$, covering several decades in flux-density. The model by Toffolatti et al. (1998), in particular, was extensively exploited to estimate the radio source contamination of CMB maps at $\mathrm{cm} / \mathrm{mm}$ wavelengths (e.g. MartínezGonzález et al. 2003; Vielva et al. 2003; Herranz et al. 2002; Refregier et al. 2000) and turns out to satisfactorily account also for the high frequency counts down to $S \simeq 20 \mathrm{mJy}$, with a maximum offset of a factor $\simeq 0.7$ but only at the brightest fluxes (cf. Fig. 13 of Bennett et al. 2003). On the other hand, the data that have been accumulating in recent years require a more detailed treatment of each of the various relevant sub-populations.

In this paper we present a new analysis encompassing canonical radio source populations as well as a variety of special classes of sources. In Sect. 2 we describe the model for canonical radio sources, allowing for different evolutionary behaviours for two classes of flat-spectrum sources (flat-spectrum radio quasars and BL Lac objects) and for steepspectrum sources, while in Sect. 3 we present the data sets used to determine the parameters. In Sect. 4 we work out predictions of counts for star-forming galaxies, extreme GPS sources, ADAF/ADIOS sources, Sunyaev-Zeldovich effects on scales from clusters of galaxies to large galaxies, radio afterglows of $\gamma$-ray bursts. Our main conclusions are summarized and discussed in Sect. 5.

We have adopted a flat $\Lambda \mathrm{CDM}$ cosmology with $\Omega_{\Lambda}=0.7$ and $H_{0}=65 \mathrm{~km} \mathrm{~s}^{-1} \mathrm{Mpc}^{-1}$.

\section{The evolutionary model for canonical radio source populations}

We have considered different epoch-dependent luminosity functions (LFs) for two flat-spectrum (flat-spectrum radio quasars (FSRQs) and BL Lacertae type objects (BL Lacs)), and for steep-spectrum radio source populations. The study by Sadler et al. (2002) of the local radio luminosity function at $1.4 \mathrm{GHz}$ yielded separate estimates for sources powered by nuclear activity (AGN) and for star-forming galaxies. By "steepspectrum sources" we mean here only the former population; the latter is considered in Sect. 4.1.

We have adopted comoving LFs (in units of $\left.\mathrm{Mpc}^{-3}(\mathrm{~d} \log L)^{-1}\right)$ of the form

$\Phi(L, z)=\frac{n_{0}}{\left(L / L_{*}\right)^{a}+\left(L / L_{*}\right)^{b}}$.

Only in the case of FSRQs enough information is available to look (although in a simplified manner) for evidences of a decline of the space density at high redshifts. For these sources, we have assumed:

$L_{*, \operatorname{FSRQ}}(z)=L_{*}(0) 10^{k_{\mathrm{ev}} z\left(2 z_{\mathrm{top}}-z\right)}$.

For the other evolving populations we have used the simpler expression:

$L_{*}(z)=L_{*}(0) \exp \left[k_{\mathrm{ev}} \tau(z)\right]$,

where $\tau(z)$ is the look-back time in units of the Hubble time, $H_{0}^{-1}$. No evolution was assumed for the lowest luminosity steep-spectrum sources $\left(\log L_{5 \mathrm{GHz}}\left(\mathrm{erg} \mathrm{s}^{-1} \mathrm{~Hz}^{-1}\right)<30.5\right)$.

We have allowed for the high-frequency spectral steepenings of steep-spectrum radio sources exploiting the quadratic fits by FR-type and radio power derived by Jackson \& Wall (2001; their Table 2). Simple power-law spectra with index $\alpha_{\text {flat }}=-0.1\left(S_{v} \propto v^{\alpha}\right)$, consistent with the results by Ricci et al. (2004), have been adopted for flat-spectrum sources.

The best fit values of the parameters were derived using the routine "amoeba" (Press et al. 1992) exploiting the downhill simplex method in multidimensions. The fitted data sets are described below and the parameter values are listed in Table 1.

\section{Data sets}

\subsection{The first year WMAP catalog of extragalactic sources}

As illustrated by Fig. 1, the first year WMAP catalog of extragalactic sources (Bennett et al. 2003) appears to be complete 
Table 1. Best fit values of the parameters of the evolutionary models for canonical radio sources.

\begin{tabular}{lccccccc}
\hline \hline Source type & \multicolumn{9}{c}{ Luminosity function } & & \multicolumn{2}{c}{ Evolution } \\
\cline { 2 - 5 } \cline { 5 - 6 } & $\log n_{0}\left(\mathrm{Mpc}^{-3}\right)$ & $a$ & $b$ & $\log L_{*}\left(\mathrm{erg} \mathrm{s}^{-1} \mathrm{~Hz}^{-1} @ 5 \mathrm{GHz}, z=0\right)$ & & $k_{\mathrm{ev}}$ & $z_{\text {top }}$ \\
\hline FSRQ & -8.988 & 0.651 & 2.888 & 34.043 & & 0.224 & 2.252 \\
BL Lac & -7.921 & 0.977 & 1.266 & 32.789 & & 0.745 & \\
Steep & -8.030 & 1.071 & 3.054 & 33.329 & & 4.902 & \\
\hline
\end{tabular}

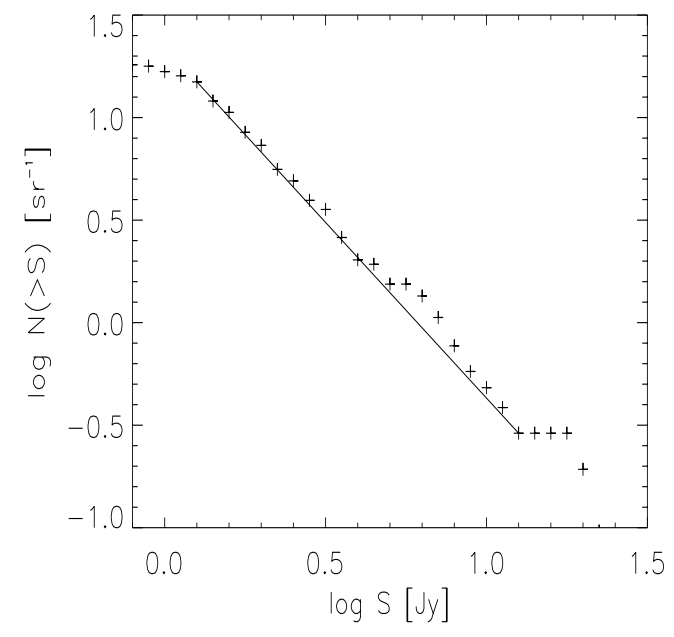

Fig. 1. Integral counts of extragalactic sources in the WMAP $K$-band (centered at $22.8 \mathrm{GHz}$ ).

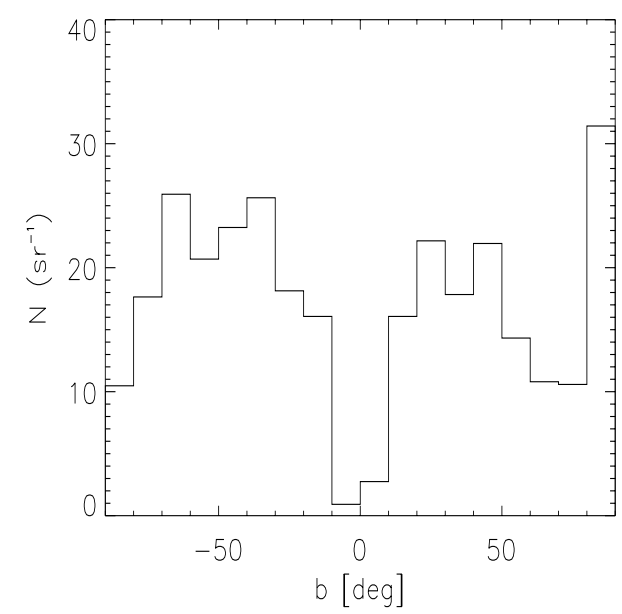

Fig. 2. Surface density of WMAP extragalactic sources as a function of Galactic latitude.

to $S_{K} \simeq 1.25 \mathrm{Jy}$ in the $K$-band, centered at $22.8 \mathrm{GHz}$ (see also Argüeso et al. 2003). Their distribution as a function of Galactic latitude (Fig. 2) shows a clear deficit at $|b|<10^{\circ}$. Removing the 4 sources in this region, we are left with 155 sources above the completeness limit, over an area of 10.4 sr. For all these sources we have determined the $4.85-22.8 \mathrm{GHz}$ spectral index, using the 4.85 flux densities from the GB6 (Gregory et al. 1996) or PMN (available at http://www.parkes.atnf.csiro.au/) catalogs. We have searched the NED and VizieR on-line databases for optical identifications and redshifts. The search yielded 91 flat-spectrum radio quasars (FSRQs), 28 BL Lacs,



Fig. 3. Redshift distribution of WMAP FSRQs (histogram) compared with the best-fit model (solid line).



Fig. 4. Differential counts of WMAP FSRQs compared with the bestfit model (solid line).

17 unclassified flat-spectrum sources and 19 steep-spectrum sources (we define as flat-spectrum sources those with spectral index $\left.\alpha>-0.5, S_{v} \propto v^{\alpha}\right)$. Redshift measurements were found for 86 FSRQs, 27 BL Lacs, 9 of the unclassified sources flat-spectrum sources and for 18 steep-spectrum sources. The redshift distributions and the counts of FSRQs (Figs. 3 and 4) and of BL Lacs have been corrected by a factor of $(1+17 / 119)$ to include the 17 unclassified flat-spectrum sources, partitioned among the two populations in proportion to the number of classified sources in each population. In computing the model redshift distributions, the surveyed area has been decreased by a factor of 0.94 in the case of FSRQs and of 0.96 in the case of 
BL Lacs, to allow for the incompleteness of redshift measurements. Poisson errors (Gehrels 1986) have been adopted both for the counts and the redshift distributions.

\subsection{The Parkes quarter-Jansky flat-spectrum sample}

The sample (Jackson et al. 2002) comprises 878 sources with spectral index $\alpha_{2.7 \mathrm{GHz}}^{5 \mathrm{GHz}} \leq-0.4$, selected from several surveys to different depths at $2.7 \mathrm{GHz}$. The catalog compiled by Jackson et al. (2002) includes optical identifications and redshifts for most of the sources; 827 are compact extragalactic radio sources, 38 have either extended radio structure or are Galactic objects, 13 are unidentified (4 of which because of obscuration by Galactic stars). Of the 827 compact extragalactic radio sources, 624 are FSRQs, 54 are BL Lacs and 149 flatspectrum radio sources. As in the case of WMAP data, we have subdivided the latter plus the unidentified sources among the FSRQs and the BL Lacs, in proportion to the fraction of classified sources belonging to each population, i.e. we increased the counts of both populations by a factor $1+162 /(624+54)$.

While the full sample has been used to derive the differential source counts (shown in Figs. 6 and 7), for the analysis of the redshift distribution we have defined a complete subsample aiming at maximizing the fraction of sources with measured redshift. To this end, we have first focussed on areas surveyed to at least $0.25 \mathrm{Jy}$, keeping only sources brighter than this limit, for a total of 618 sources. Of these, 437 are FSRQs, 360 of which (82\%) have measured redshift, 47 are BL Lacs, only 10 of which $(21 \%)$ have measured redshift, and 111 are flatspectrum galaxies (32, i.e. $29 \%$, with redshift). Clearly, only in the case of FSRQs the fraction of sources with measured redshifts is large enough for a meaningful redshift distribution to be derived. Further restricting the sample to $\delta>-50^{\circ}$, we are left with 370 FSRQs, 345 of which (93\%) with measured redshift, 47 BL Lacs, 95 sources classified as galaxies and 2 unidentified sources. The redshift distribution of FSRQs for this sub-sample is thus well defined (Fig. 8). As in the case of the counts, we have corrected the redshift distribution by a factor of $1+(95+2) /(370+47)$ to allow for a fraction of flatspectrum galaxies and of unidentified sources proportional to the ratio FSRQ/(FSRQ+BL Lacs).

\subsection{The Kühr 1 Jy sample}

The sample (Kühr et al. 1981) comprises 518 sources to a $5 \mathrm{GHz}$ flux density limit of $1 \mathrm{Jy}$, over an area of $9.811 \mathrm{sr}$. Based on the catalogued spectral indices, 299 sources are flatspectrum $\left(\alpha>-0.5, S_{\nu} \propto v^{\alpha}\right)$ and 219 are steep-spectrum. The former population includes 212 FSRQs, 200 of which (94\%) have measured redshift, 26 BL Lacs (20 of which, 77\%, with measured redshift, and 61 either classified as galaxies or missing a morphological classification. As before, we have distributed the latter sources among FSRQs and BL Lacs, thus increasing the counts of both types by a factor of $1+61 /(212+26)$. The fit of the redshift distribution of FSRQs is shown in Fig. 9.



Fig. 5. Comparison of the model (solid line) with the differential $15 \mathrm{GHz}$ counts from the main survey (filled circles) and the deeper survey (filled squares) by Waldram et al. (2003) and with total differential counts at $18 \mathrm{GHz}$ by Ricci et al. (2004; crosses).

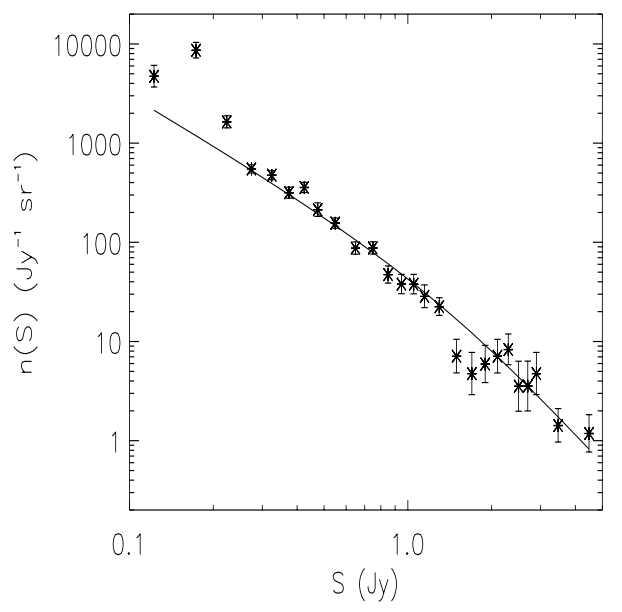

Fig. 6. Counts of FSRQs in the Parkes quarter-Jy sample compared with the best-fit model (solid line).

\subsection{Other data sets}

Furthermore, we have fitted the $5 \mathrm{GHz}$ counts (Fig. 10) by Gregory \& Condon (1991) and Pauliny-Toth et al. (1978a,b), the $15 \mathrm{GHz} 9 \mathrm{C}$ counts plus the $18 \mathrm{GHz}$ ATCA counts (Fig. 5) by Waldram et al. (2003) and Ricci et al. (2004), respectively, and the ATCA $18 \mathrm{GHz}$ counts (Fig. 11) of flat-spectrum sources (Ricci et al. 2004).

In our scheme, blazars (FSRQs plus BL Lacs) account for the entire population of flat-spectrum radio sources. We thus require that the sum of their local luminosity functions matches the estimates of the local luminosity function of flat-spectrum sources by Peacock (1985) and Toffolatti et al. (1987). The fit is shown in Fig. 12.

\section{Special source populations}

\subsection{Star-forming galaxies}

The radio emission of galaxies correlates with their starformation rate, as demonstrated by the well established tight 


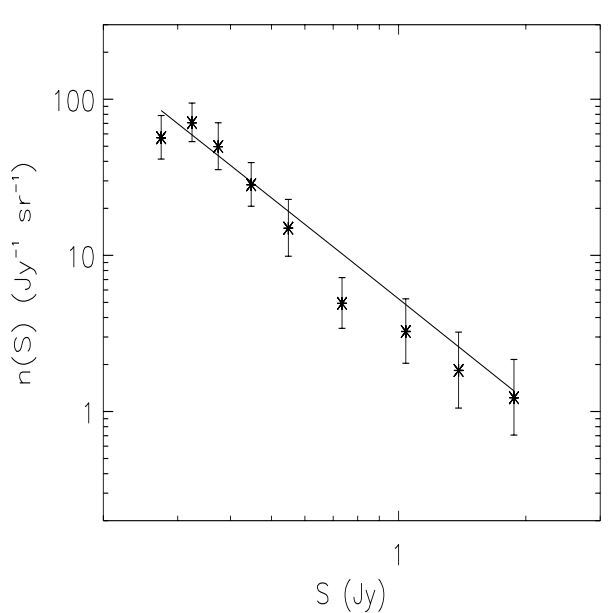

Fig. 7. Counts of BL Lac objects in the Parkes quarter-Jy sample compared with the best-fit model (solid line).

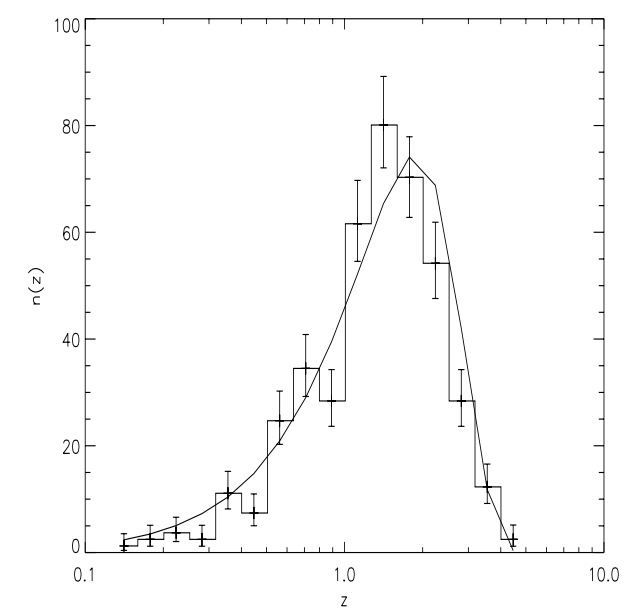

Fig. 8. Redshift distribution of FSRQs in the Parkes quarter-Jy sample (histogram) compared with the best-fit model (solid line).

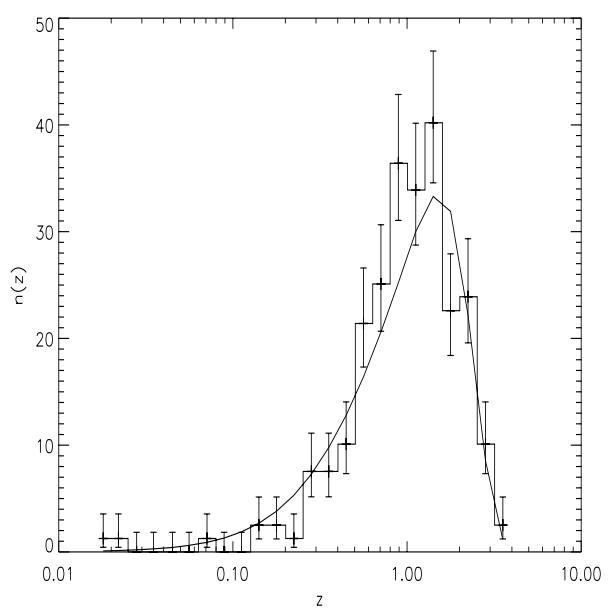

Fig. 9. Redshift distribution of FSRQs in the Kühr et al. (1981) sample (histogram) compared with the best-fit model (solid line).

correlation with far-IR emission (Helou et al. 1985; Gavazzi et al. 1986; Condon 1992; Garrett 2002; Morganti et al. 2004). The cross-over between synchrotron plus free-free emission,

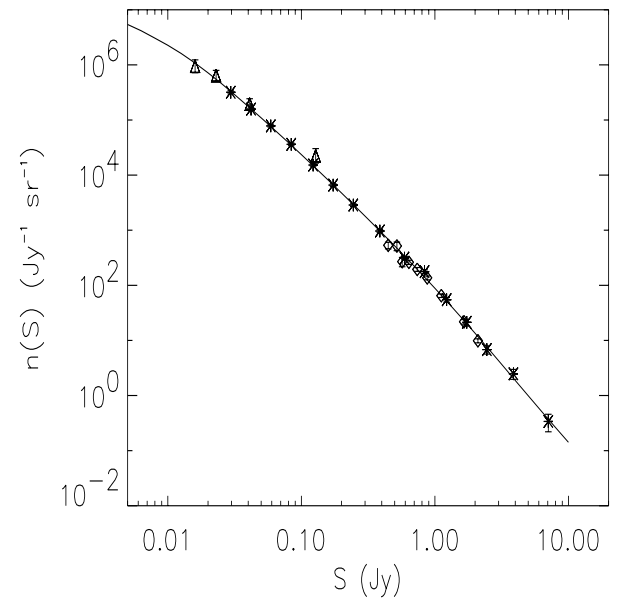

Fig. 10. Differential counts at $5 \mathrm{GHz}$ compared with the best-fit model (solid line). Data from Gregory \& Condon (1991, asterisks) and Pauliny-Toth et al. (1978a, diamonds; 1978b, triangles).



Fig. 11. Differential counts at $18 \mathrm{GHz}$ of flat-spectrum sources by Ricci et al. (2004) compared with the best-fit model (solid line).

prevailing at $\mathrm{cm}$ wavelengths, and thermal dust emission generally occurs at $\lambda \simeq 2-3 \mathrm{~mm}$, so that at frequencies of tens of $\mathrm{GHz}$ there are contributions from both components, the former being associated to normal late-type and starburst galaxies at low to moderate redshifts, the latter to the high-redshift population detected by sub-mm surveys ("SCUBA galaxies").

Following Granato et al. (2001, 2004) we interpret "SCUBA galaxies" as proto-spheroidal galaxies in the process of forming most of their stars. Their epoch-dependent luminosity function is computed using the physical model by Granato et al. (2004), which describes the star-formation and chemical enrichment histories and, interfaced with the code GRASIL (Silva et al. 1998), yields the spectrophotometric evolution from radio to X-ray wavelengths. The values of GRASIL parameters were determined by Silva et al. (2004) comparing the model predictions with a broad variety of observational data.

Normal late-type and starburst galaxies are dealt with in a more phenomenological manner, following Silva et al. (2004). Briefly, we adopted the $60 \mu \mathrm{m}$ local luminosity functions of "warm" and "cold" galaxies (based on IRAS colors), determined by Saunders et al. (1990), for starburst and late-type 


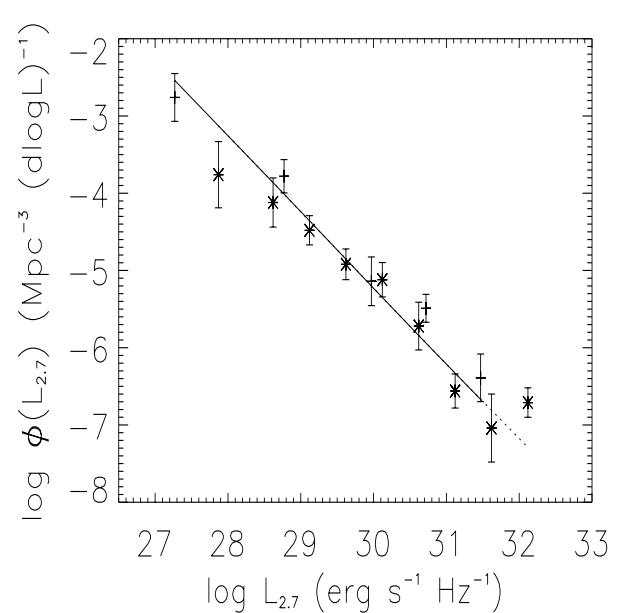

Fig. 12. Local luminosity functions at $2.7 \mathrm{GHz}$ by Peacock (1985) (crosses) and Toffolatti et al. (1987) (asterisks) compared with the best-fit model (solid line).

galaxies, respectively. The local luminosity functions were cut off at $L_{60 \mu \mathrm{m}}=2 \times 10^{32} \mathrm{erg} / \mathrm{s} / \mathrm{Hz}$. Extrapolations to other wavelengths were made using GRASIL spectral energy distributions fitting the data on NGC 6090 (for starburst galaxies) and NGC 6946 (for late-type galaxies).

A density and luminosity evolution model $(L F[L(z), z]=$ $\left.L F\left[L(z) /(1+z)^{2.5}, z=0\right] \times(1+z)^{3.5}\right)$ was adopted for starburst galaxies, while a mild pure luminosity evolution $(L(z)=$ $\left.L(0)(1+z)^{1.5}\right)$ was assumed for normal late-type galaxies. These evolutionary laws were applied up to $z=1$. The luminosity functions were then kept to the (comoving) values they have at $z=1$ up to a redshift cut-off $z_{\text {cutoff }}=1.5$.

\subsection{Extreme $G H z$ peaked spectrum (GPS) sources}

A class of sources that is expected to come out in high frequency surveys is that of extreme GHz Peaked Spectrum (GPS) or very high frequency peakers. GPS sources are powerful $\left(\log P_{1.4 \mathrm{GHz}} \gtrsim 25 \mathrm{~W} \mathrm{~Hz}^{-1}\right)$, compact ( $\lesssim 1 \mathrm{kpc}$ ) radio sources with a convex spectrum peaking at $\mathrm{GHz}$ frequencies (see O'Dea 1998, for a comprehensive review). They are identified with both galaxies and quasars.

It is now widely agreed that GPS sources correspond to the early stages of the evolution of powerful radio sources, when the radio emitting region grows and expands within the interstellar medium of the host galaxy, before plunging in the intergalactic medium and becoming an extended radio source (Fanti et al. 1995; Readhead et al. 1996; Begelman 1996; Snellen et al. 2000). Conclusive evidence that these sources are young came from measurements of propagation velocities. Velocities of up to $\simeq 0.4 c$ were measured, implying dynamical ages $\sim 10^{3}$ years (Polatidis et al. 1999; Taylor et al. 2000; Tschager et al. 2000). The identification and investigation of these sources is therefore a key element in the study of the early evolution of radioloud AGNs.

There is a clear anti-correlation between the peak (turnover) frequency and the projected linear size of GPS sources (and of compact steep-spectrum sources), suggesting that the process (probably synchrotron self-absorption, although free-free absorption is also a possibility) responsible for the turnover depends simply on the source size. Although this anti-correlation does not necessarily define the evolutionary track, a decrease of the peak frequency as the emitting blob expands is indicated. Thus high-frequency surveys may be able to detect these sources very close to the moment when they turn on.

The self-similar evolution models by Fanti et al. (1995) and Begelman (1996) imply that the radio power drops as the source expands, so that GPS's evolve into lower luminosity radio sources, while their luminosities are expected to be very high during the earliest evolutionary phases, when they peak at high frequencies. De Zotti et al. (2000) showed that, with a suitable choice of the parameters, this kind of models may account for the observed counts, redshift and peak frequency distributions of the samples then available. The data indicated strongly different evolutionary properties between GPS galaxies and quasars, at variance with unification scenarios.

The models by De Zotti et al. (2000) imply that extreme GPS quasars, peaking at $v>20 \mathrm{GHz}$, comprise a quite substantial fraction of bright radio sources in the WMAP survey at $v \simeq 20 \mathrm{GHz}$, while GPS galaxies with similar $v_{\text {peak }}$ should be about 10 times less numerous. For a maximum rest-frame peak frequency $v_{p, i}=200 \mathrm{GHz}$, the model predicts about $10 \mathrm{GPS}$ quasars with $S_{30 \mathrm{GHz}}>2$ Jy peaking at $\geq 30 \mathrm{GHz}$ over the $10.4 \mathrm{sr}$ at $|b|>10^{\circ}$.

Although the number of candidate GPS quasars in the WMAP survey is consistent with that expected from the models, when data at additional frequencies (Trushkin 2003) are taken into account such candidates look more blazars caught during a flare optically thick up to high frequencies. Furthermore, Tinti et al. (2004) have shown that most, perhaps two thirds, of the quasars in the sample of High Frequency Peaker candidates selected by Dallacasa et al. (2000) are likely blazars, while all the 10 candidates classified as galaxies are consistent with being truly young sources.

Snellen et al. (2000) support a scenario whereby the luminosity of GPS sources increases with time (while the peak frequency decreases) until a linear size $\simeq 1 \mathrm{kpc}$ is reached, and decreases subsequently. Although predictions for GPS source counts have not been worked out in this framework, these objects must be very rare at bright high frequency fluxes. In Figs. 13 and 14 we have provisionally plotted (dashed line in the upper right-hand panel) the predictions of the models by De Zotti et al. (2000) with a maximum intrinsic peak frequency $v_{\mathrm{p}, i}=200 \mathrm{GHz}$, decreasing by a factor of 3 the contribution of GPS quasars.

Large area high frequency surveys, coupled with follow-up simultaneous multifrequency observations of GPS candidates, would be essential to assess the evolutionary properties of this population.

\subsection{Late stages of AGN evolution}

High radio frequency observations are also crucial to investigate late stages of the AGN evolution, characterized by low radiation/accretion efficiency. This matter was recently brought 

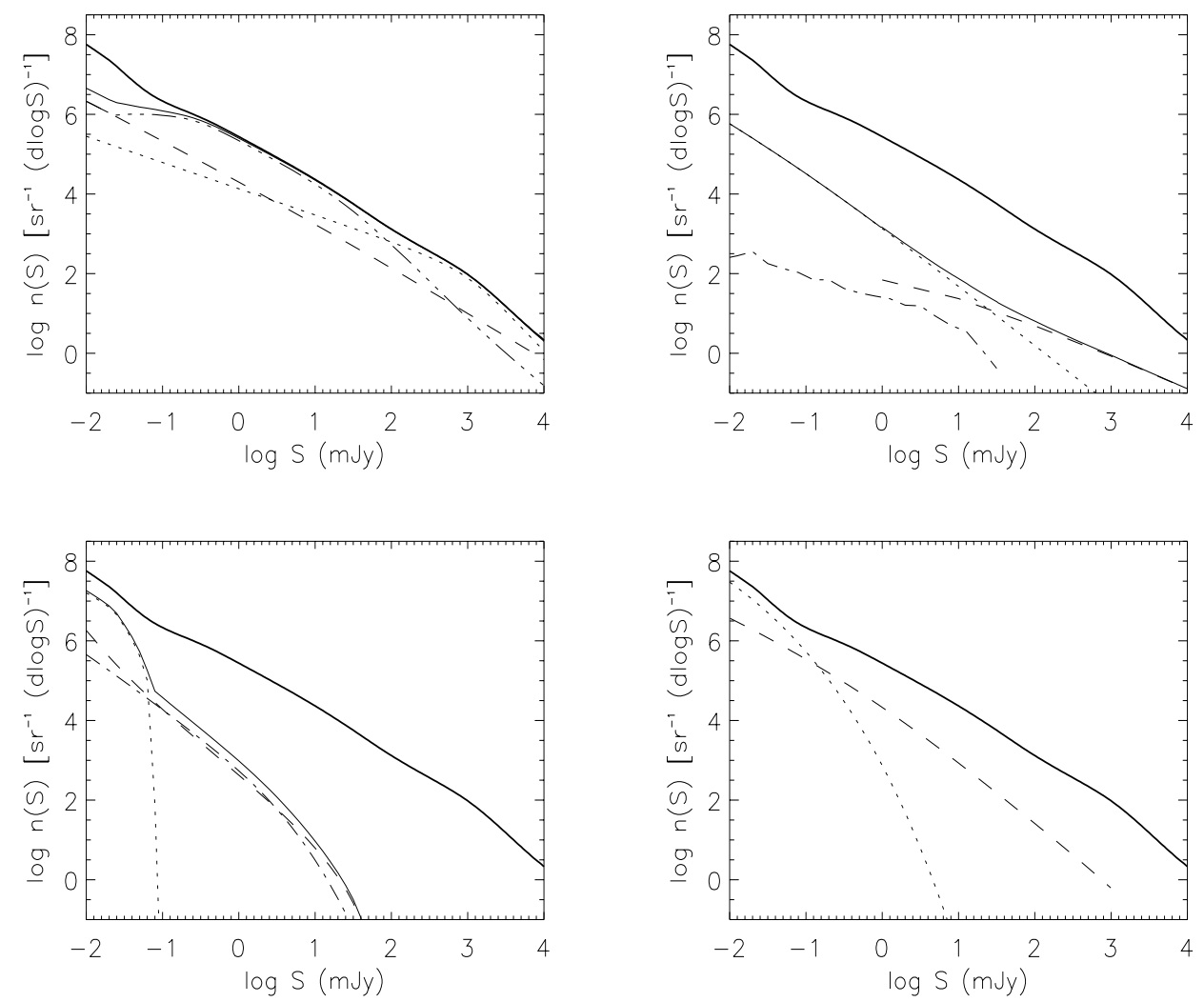

Fig. 13. Predicted differential counts at $20 \mathrm{GHz}$ for various extragalactic source populations. Upper left-hand panel (classical radio sources): FSRQs (dotted line); BL Lacs (dashed line); steep-spectrum sources (triple dot-dashed line). Upper right-hand panel (special sources): ADAFs (dotted line); extreme GPS quasars and galaxies (dashed line); GRB afterglows (dot-dashed line). Lower left-hand panel (star-forming galaxies): proto-spheroids (dotted line); spirals (dot-dashed line); starburst galaxies (dashed line). Lower right-hand panel: SZ effects on galactic scales (dotted line) and on cluster scales (dashed line). The sum of contributions shown in each panel and the overall total counts are indicated by a thin and thick solid line, respectively.

into sharper focus by the discovery of ubiquitous, moderate luminosity hard X-ray emission from nearby ellipticals. VLA studies at high radio frequencies (up to $43 \mathrm{GHz}$ ) have shown, albeit for a limited sample of objects, that all the observed compact cores of elliptical and S0 galaxies have spectra rising up to $\simeq 20-30 \mathrm{GHz}$ (Di Matteo et al. 1999).

There is growing evidence that essentially all massive ellipticals host super-massive black holes (see, e.g., Kormendy $\&$ Gebhardt 2001). Yet, nuclear activity is not observed at the level expected from Bondi's (1952) spherical accretion theory, in the presence of extensive hot gaseous halos, and for the usually assumed radiative efficiency $\simeq 10 \%$ (Di Matteo et al. 1999). However, as proposed by Rees et al. (1982), the final stages of accretion in elliptical galaxies may occur via AdvectionDominated Accretion Flows (ADAFs), characterized by a very low radiative efficiency (Fabian \& Rees 1995). The ADAF scenario implies strongly self-absorbed thermal cyclo-synchrotron emission due to a near equipartition magnetic field in the inner parts of the accretion flows, most easily detected at $\mathrm{cm}$ to $\mathrm{mm}$ wavelengths. However the ADAF scenario is not the only possible explanation of the data, and is not problem-free. Chandra X-ray observations of Sgr A, at the Galactic Center, are suggestive of a considerably lower, compared to Bondi's, accretion rate (Baganoff et al. 2003), so that the very low ADAF radiative efficiency may not be required. A stronger argument against a pure ADAF in the Galactic Center is that the emission is strongly polarized at $\mathrm{mm} / \mathrm{sub}-\mathrm{mm}$ wavelengths (Aitken et al. 2000; Agol 2000). Also Di Matteo et al. (1999, 2001) found that the high frequency nuclear radio emission of a number of nearby early-type galaxies is substantially below the predictions of standard ADAF models. The observations would be more consistent with the adiabatic inflow-outflow solutions (ADIOS), developed by Blandford \& Begelman (1999), whereby the energy liberated by the accretion drives an outflow at the polar region carrying a considerable fraction of the mass, energy and angular momentum available in the accretion flow, thus suppressing the radio emission from the inner regions. Both the intensity and the peak of the radio emission depend on the mass loss rate.

We have estimated the counts yielded by these low radiative efficiency flows (LREF) following Perna \& Di Matteo (2000), i.e. we have taken the number density of such sources to be given, as a function of redshift, by their Eq. (5), with a cut-off at $z=1$, and have used the emission spectrum corresponding to $p=0.2$ in their Fig. 1, which corresponds to the upper end of the range of observed luminosities. The counts we obtain, shown by the dotted line in the upper right-hand panel of Figs. 13 and 14, are about a factor of ten below the estimates by Perna \& Di Matteo (2000). We do not understand the reason for this strong discrepancy. We note however, that the 



Fig. 14. Same as in Fig. 13, but at $30 \mathrm{GHz}$.

conclusion of these Authors that the $30 \mathrm{GHz}$ LREF counts can be comparable or even higher than those of sources known from low-frequency surveys is ruled out by the results of the Ryle telescope, ATCA and WMAP surveys.

\section{Sunyaev-Zeldovich effects}

The Sunyaev \& Zel'dovich (SZ) effect (Sunyaev \& Zeldovich 1972) arises due to the inverse Compton scatter of CMB photons against the hot electrons. The CMB intensity change is given by:

$\Delta I_{v}=2 \frac{\left(k T_{\mathrm{CMB}}\right)^{3}}{(h c)^{2}} y g(x)$

where $T_{\mathrm{CMB}}$ is the CMB temperature and $x=h v / k T_{\mathrm{CMB}}$.

The spectral form of this "thermal effect" is described by the function

$g(x)=x^{4} \mathrm{e}^{x}[x \cdot \operatorname{coth}(x / 2)-4] /\left(\mathrm{e}^{x}-1\right)^{2}$,

which is negative (positive) at values of $x$ smaller (larger) than $x_{0}=3.83$, corresponding to a critical frequency $v_{0}=217 \mathrm{GHz}$.

The Comptonization parameter is

$y=\int \frac{k T_{\mathrm{e}}}{m c^{2}} n_{\mathrm{e}} \sigma_{\mathrm{T}} \mathrm{d} l$

where $n_{\mathrm{e}}$ and $T_{\mathrm{e}}$ are the electron density and temperature, respectively, $\sigma_{\mathrm{T}}$ is the Thomson cross section, and the integral is over a line of sight through the plasma.

With respect to the incident radiation field, the change of the CMB intensity across a cluster can be viewed as a net flux
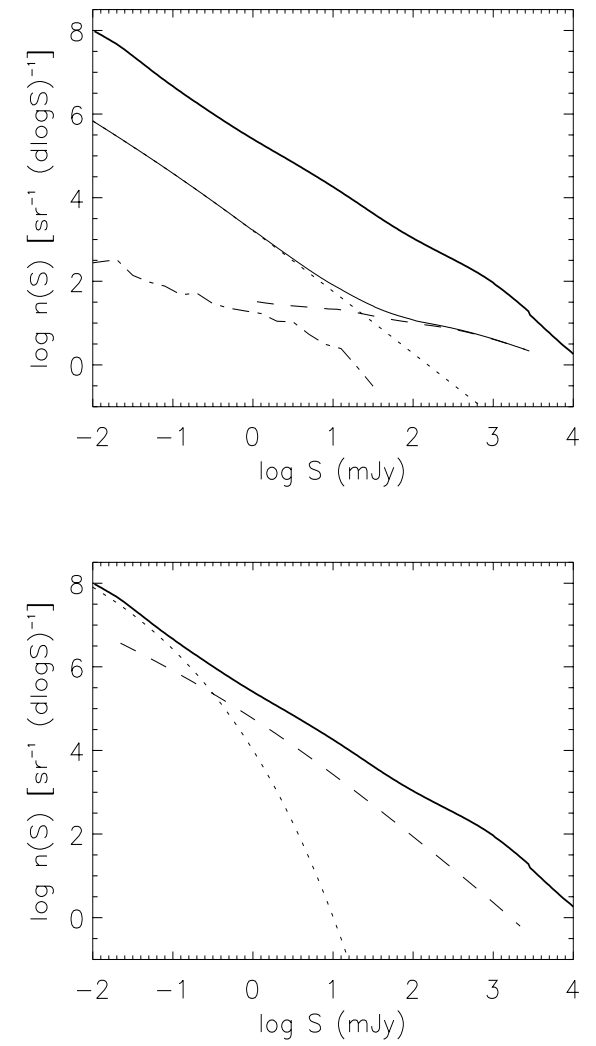

emanating from the plasma cloud, given by the integral of intensity change over the solid angle subtended by the cloud:

$\Delta F_{v}=\int \Delta I_{v} \mathrm{~d} \Omega \propto Y \equiv \int y \mathrm{~d} \Omega$.

In the case of hot gas trapped in the potential well due to an object of total mass $M$, the parameter $Y$ in Eq. (7), called integrated $Y$-flux, is proportional to the gas-mass-weighted electron temperature $\left\langle T_{\mathrm{e}}\right\rangle$ and to the gas mass $M_{\mathrm{g}}=f_{\mathrm{g}} M$ :

$Y \propto f_{\mathrm{g}}\left\langle T_{\mathrm{e}}\right\rangle M$

At the frequencies considered here, the $Y$-flux is negative and can therefore be distinguished from the positive signals due to the other source populations.

\subsection{Sunyaev-Zeldovich effects in galaxy clusters}

The SZ effect from the hot gas responsible for the X-ray emission of rich clusters of galaxies has been detected with high signal to noise and even imaged in several tens of objects (Carlstrom et al. 2002).

Recent X-ray Chandra observations show that for massive clusters the gas fraction is remarkably constant, $f_{\mathrm{g}}=$ $0.113 \pm 0.005$, independently of the cluster redshift (Allen et al. 2002). Simple scaling laws are used to derive, under the hydrostatic equilibrium hypothesis, the relation between the mass and the temperature of a cluster (see, e.g., Barbosa et al. 1996; 
Colafrancesco et al. 1997; Bryan \& Norman 1998, and references therein):

$T(M, z)=T_{15} h^{2 / 3}\left[\frac{\Omega_{0} \Delta_{\mathrm{c}}\left(\Omega_{0}, z\right)}{180}\right]^{1 / 3}\left(\frac{M}{M_{15}}\right)^{2 / 3}(1+z)$,

where $\Delta_{c}$ is the non-linear density contrast of a cluster that collapsed at redshift $z$ and $T_{15}$ is the temperature of a cluster of mass $M_{15}=10^{15} h^{-1} M_{\odot}$ which collapses today. We calculate $\Delta_{c}$ using the equations reported in the appendix of Colafrancesco et al. (1997): the $M-T$ relation calculated in this way is perfectly consistent with Eq. (9) of Bryan \& Norman (1998). Both simulations and observations indicate that temperatures of massive clusters follow quite well the relationship with mass and redshift expressed by Eq. (9) (see, e.g., Ettori et al. 2004, and references therein). Nevertheless, there is still some discussion on the exact value of $T_{15}$, whose values range from $6.6 \mathrm{keV}$ to $8.8 \mathrm{keV}$ (see, e.g., Pierpaoli et al. 2001, and references therein). This represents, at the moment, the largest source of uncertainty for the normalization of the power spectrum of the matter density fluctuation $P(k) \equiv\left|\delta_{k}\right|^{2}$ inferred from the observed cluster X-ray temperature function (XTF). Such normalization is usually given in term of the value of $\sigma_{8}$, i.e. the variance of the density perturbation $\sigma^{2}(R, z) \propto \int_{o}^{\infty} k^{3} P(k, z) W^{2}(k R) \mathrm{d} k / k$, where $W(k R)$ is the windows function corresponding to the smoothing of the density field (see, e.g., Peebles 1993), on the scale of $R=8 h^{-1} \mathrm{Mpc}$. Lower values of $T_{15}$ result in higher values of $\sigma_{8}$ and viceversa (see, e.g., Ikebe et al. 2002; Pierpaoli et al. 2003). Here we choose $T_{15}=7.75 \mathrm{keV}$, consistent with the simulations of Eke et al. (1998), and $\sigma_{8}=0.85$ yielding an XTF consistent with the observed one (see, e.g., Pierpaoli et al. 2003). The number counts for SZ clusters are then given by:

$N\left(>\overline{\Delta F_{v}}\right)=\int \frac{\mathrm{d} V}{\mathrm{~d} z} \mathrm{~d} z \int_{\bar{M}\left(\overline{\Delta F_{v}}, z\right)} \mathrm{d} M N(M, z)$,

where $N(M, z)$ is the cluster mass function and the lower bound of the mass integral is determined by requiring that clusters of mass $M>\bar{M}$ at redshift $z$ have SZ fluxes greater than $\overline{\Delta F_{v}}$ (for details see, e.g., Colafrancesco et al. 1997). Here we adopt the mass function of Sheth et al. (2001):

$$
\begin{aligned}
N(M, z) \mathrm{d} M= & \sqrt{\frac{2 a A^{2}}{\pi}} \rho_{0} \frac{1}{M^{2}}\left[1+\left(\frac{\sigma(M, z)}{\sqrt{a} \delta_{c}}\right)^{2 p}\right] \\
& \times \frac{\mathrm{d} \ln \sigma}{\operatorname{dln} M} \frac{\delta_{c}}{\sigma(M, z)} \exp \left[-\frac{1}{2} \frac{a \delta_{c}^{2}}{\sigma^{2}(M, z)}\right],
\end{aligned}
$$

where $\rho_{0}$ is the present-day mass density of the Universe, $\delta_{c}=$ 1.68, $a=0.707, A=0.3222, p=0.3$.

We caution that the 20 and $30 \mathrm{GHz}$ counts of cluster SZ effects shown in the lower right-hand panel of Figs. 13 and 14 could be substantially affected by radio-source contamination since radio sources are strongly correlated with clusters of galaxies (Holder 2002; Massardi \& De Zotti 2004). Although the available data do not allow a quantitative estimate of the effect on counts, we note that a single powerful radio source can fill in the SZ decrement of a small group of galaxies.

\subsection{Galaxy-scale Sunyaev-Zeldovich effects}

Evidences of statistically significant detections at $30 \mathrm{GHz}$ of arcminute scale fluctuations well in excess of predictions for primordial anisotropies of the cosmic microwave background (CMB) have recently been obtained by the CBI (Mason et al. 2003) and BIMA (Dawson et al. 2002) experiments. The interpretation of these results is still debated. Extragalactic sources are potentially the dominant contributor to fluctuations on these scales and must be carefully subtracted out. And indeed both groups devoted a considerable effort for this purpose. However a quite substantial residual contribution to the CBI signal is difficult to rule out (De Zotti et al. 2004; Holder 2002).

If indeed the detected signal cannot be attributed to extragalactic radio sources, its most likely source is the thermal Sunyaev-Zeldovich (SZ) effect (Gnedin \& Jaffe 2001). The small-scale fluctuations due to the SZ within rich clusters of galaxies has been extensively investigated (Komatsu \& Kitayama 1999; Bond et al. 2002). The estimated power spectrum was found to be very sensitive to the normalization $\left(\sigma_{8}\right)$ of the density perturbation spectrum. A $\sigma_{8} \gtrsim 1$, somewhat higher than indicated by other data sets, is apparently required to account for the CBI data.

On the other hand, significant SZ signals may be associated to the formation of spheroidal galaxies (De Zotti et al. 2004; Rosa-Gonzalez et al. 2004). The proto-galactic gas is expected to have a large thermal energy content, leading to a detectable SZ signal, both when the proto-galaxy collapses with the gas shock-heated to the virial temperature (Rees \& Ostriker 1977; White \& Rees 1978), and in a later phase as the result of strong feedback from a flaring active nucleus (Ikeuchi 1981; Natarajan et al. 1998; Natarajan \& Sigurdssson 1999; Aghanim et al. 2000; Platania et al. 2002; Lapi et al. 2003).

These SZ signals, showing up on sub-arcmin scales, are potentially able to account for the BIMA results. Proto-galactic gas heated at the virial temperature and with a cooling time comparable with the expansion time may provide the dominant contribution. It must be stressed, however, that estimates are plagued by large uncertainties reflecting our poor understanding of the complex physics governing the thermal history of the proto-galactic gas, and, as stressed by the authors themselves, the counts of galactic scale SZ effects computed by De Zotti et al. (2004) may be more conservatively viewed as upper limits. Furthermore, the radio emission from the galaxy can partially fill in the SZ dip, particularly if the galaxy hosts a radio active nucleus (Platania et al. 2002).

On the other hand, the SZ effect turns out to be an effective probe of the thermal state of the gas and of its evolution, so that its detection, that may be within reach of future high frequency surveys (cf. the lower right-hand panel of Figs. 13 and 14) would provide unique information on early phases of galaxy evolution, essentially unaccessible by other means.

\subsection{Radio afterglows of $\gamma$-ray bursts}

The afterglow emission of GRBs can be modelled as synchrotron emission from a decelerating blast wave in an ambient medium, plausibly the interstellar medium of the host galaxy 
(Waxman 1997; Wijers \& Galama 1999; Meszaros 1999). The radio flux, above the self-absorption break occurring at $\$ 5 \mathrm{GHz}$, is proportional to $v^{1 / 3}$ up to a peak frequency that decreases with time. In the upper right-hand panel of Figs. 13 and 14 we show (dot-dashed line) the estimates of the counts of GRB afterglows (kindly provided by B. Ciardi) yielded by the Ciardi \& Loeb (2000) model. According to these estimates, a large area survey at $\simeq 1 \mathrm{~cm}$ to a flux limit $\simeq 1 \mathrm{mJy}$ should discover some GRBs (see also Seaton \& Partridge 2001).

\section{Discussion and conclusions}

A synoptic view of the contributions of the above source populations to the 20 and $30 \mathrm{GHz}$ differential counts is presented in Figs. 13 and 14. As expected, and directly demonstrated by the high frequency 9C (Taylor et al. 2001; Waldram et al. 2003) and ATCA (Ricci et al. 2004) surveys, the dominant population at bright flux densities are blazars. These are, however, a composite population, including the rapidly evolving Flat Spectrum Radio Quasars and the slowly evolving BL Lac objects. The former sub-population is prominent above $\simeq 100 \mathrm{mJy}$, but its counts converge rapidly at fainter flux densities, eventually falling below those of steep-spectrum sources and approaching those of BL Lacs.

Figures 13 and 14 also show that ongoing high frequency surveys are not far from serendipitous observations of cluster SZ effects, easily recognizable because they show up as negative peaks.

It is well known from very deep surveys at $1.4-8.4 \mathrm{GHz}$ that a large fraction of micro-Jy radio sources are moderate redshift active star forming galaxies (Gruppioni et al. 2001; Fomalont et al. 2002; Chapman et al. 2003). Our models are consistent with these results, and indicate that below a few tens of $\mu \mathrm{Jy}$ (at 20-30 GHz), there is a further change in the composition of the radio-source population as proto-spheroidal galaxies come into play, suddenly increasing the ratio of star forming to classical radio sources. Since these objects are predicted to be at typical redshifts of 2-3 and to be highly obscured by dust, there appearance will also increase the fraction of optically very faint sources and strongly shift to higher redshifts the redshift distribution.

The emission of star forming galaxies in the spectral region of interest here is dominated by synchrotron and free-free processes at rest-frame wavelengths longer than a few $\mathrm{mm}$, and by thermal dust radiation at shorter wavelengths. The latter emission is characterized by a steeply inverted spectrum, $S_{v} \propto v^{\alpha}$, with $\alpha \sim 4$, causing a strongly negative $K$-correction and a sharp steepening of the counts. Counts of proto-spheroidal galaxies could be significantly higher than shown in Figs. 13 and 14 if the mm excess detected in our own Galaxy, combining Archeops with WMAP and DIRBE data (Bernard et al. 2003; Dupac et al. 2003), and in NGC 1569 is a general property of the SED of dusty galaxies.

Below $\simeq 100 \mu \mathrm{Jy}$ a significant contribution to the 20 $30 \mathrm{GHz}$ counts may come from Sunyaev-Zeldovich (1972) signals (De Zotti et al. 2004; Rosa-Gonzalez et al. 2004) or freefree emission (Oh 1999) produced by proto-galactic plasma.
While no attempts to estimate the counts of proto-galactic freefree sources have been produced yet, we show in Figs. 13 and 14 the tentative estimates of the counts of SZ effects by De Zotti et al. (2004), that, as pointed out by the authors, should be more conservatively interpreted as (possibly generous) upper limits.

Acknowledgements. We thank Benedetta Ciardi for having kindly provided the counts of radio afterglows of $\gamma$-ray bursts. This research was partly supported by the Italian Space Agency (ASI) and by the Italian MIUR through a COFIN grant. DM acknowledges partial financial support from the Fondazione "G. Tovini", and LT and JGN from the Spanish MEC, project ESP2002-04141-C03-01.

\section{References}

Aghanim, N., Balland, C., \& Silk, J. 2000, A\&A, 357, 1

Agol, E. 2000, ApJ, 538, L121

Aitken, D. K., Greaves, J., Chrysostomou, A., et al. 2000, ApJ, 534, L173

Allen, S. W., Schmidt, R. W., \& Fabian, A. C. 2002, MNRAS, 334, L11

Argüeso, F., González-Nuevo, J., \& Toffolatti, L. 2003, ApJ, 598, 86

Baganoff, F. K., Maeda, Y., Morris, M., et al. 2003, ApJ, 591, 891

Barbosa, D., Bartlett, J. G., Blanchard, A., \& Oukbir, J. 1996, A\&A, 314,13

Begelman, M. C. 1996, in Cygnus A - Study of a Radio Galaxy, ed. C. L. Carilli, \& D. E. Harris (Cambridge University Press), 209

Bennett, C. L., Hill, R. S., Hinshaw, G., et al. 2003, ApJS, 148, 97

Blandford, R. D., \& Begelman, M. C. 1999, MNRAS, 303, L1

Bernard, J.-Ph., Meny, C., Dupac, X., et al. 2003, paper presented at the Planck WG7 Meeting, Jodrell Bank

Bond, J. R., Contaldi, C. R., Pen, U. L., et al. 2002, ApJ, submitted [arXiv: astro-ph/0205386]

Bondi, H. 1952, MNRAS, 112, 195

Bryan, G. L., \& Norman, M. L. 1998, ApJ, 495, 80

Carlstrom, J. E., Holder, G. P., \& Reese, E. D. 2002, ARA\&A, 40, 643

Chapman, S. C., Barger, A. J., Cowie, L. L., et al. 2003, ApJ, 585, 57

Ciardi, B., \& Loeb, A. 2000, ApJ, 540, 687

Colafrancesco, S., Mazzotta, P., Rephaeli, Y., \& Vittorio, N. 1997, ApJ, 479, 1

Condon, J. J. 1992, ARA\&A, 30, 575

Dallacasa, D., Stanghellini, C., Centonza, M., \& Fanti, R. 2000, A\&A, 363,887

Dawson, K. S., Holzapfel, W. L., Carlstrom, J. E., et al. 2002, ApJ, 581,86

De Zotti, G., Burigana, C., Cavaliere, A., et al. 2004, in proc. int. symp. Plasmas in the Laboratory and in the Universe: new insights and new challenges, ed. G. Bertin, D. Farina, \& R. Pozzoli, AIP Conf. Proc., 703, 375

De Zotti, G., Granato, G. L., Silva, L., Maino, D., \& Danese, L. 2000, A\&A, 354, 467

Di Matteo, T., Carilli, C. L., \& Fabian, A. C. 2001, ApJ, 547, 731

di Matteo, T., Fabian, A. C., Rees, M. J., Carilli, C. L., \& Ivison, R. J. 1999, MNRAS, 305, 492

Dunlop, J. S., \& Peacock, J. A. 1990, MNRAS, 247, 19

Dupac, X., Bernard, J.-P., Boudet, N., et al. 2003, A\&A, 404, L11

Eke, V. R., Navarro, J. F., Frenk, C. S. 1998, ApJ, 503, 569

Ettori, S., Tozzi, P., Borgani, S., \& Rosati, P. 2004, A\&A, 417, 13

Fabian, A. C., \& Rees, M. J. 1995, MNRAS, 277, L55

Fanti, C., Fanti, R., Dallacasa, D., et al. 1995, A\&A, 302, 317

Fomalont, E. B., Kellermann, K. I., Partridge, R. B., Windhorst, R. A., \& Richards, E. A. 2002, AJ, 123, 2402 
Galliano, F., Madden, S. C., Jones, A. P., et al. 2003, A\&A, 407, 159

Garrett, M. A. 2002, A\&A, 384, L19

Gavazzi, G., Cocito, A., \& Vettolani, G. 1986, ApJ, 305, L15

Gehrels, N. 1986, ApJ, 303, 336

Gnedin, N. Y., \& Jaffe, A. H. 2001, ApJ, 551, 3

Grainge, K., Carreira, P., Cleary, K., et al. 2003, MNRAS, 341, L23

Granato, G. L., De Zotti, G., Silva, L., Bressan, A., \& Danese, L. 2004, ApJ, 600, 580

Granato, G. L., Silva, L., Monaco, P., et al. 2001, MNRAS, 324, 757

Gregory, P. C., \& Condon, J. J. 1991, ApJS, 75, 1011

Gregory, P. C., Scott, W. K., Douglas, K., \& Condon, J. J. 1996, ApJS, 103,427

Gruppioni, C., Oliver, S., \& Serjeant, S. 2001, Ap\&SS, 276, 791

Helou, G., Soifer, B. T., \& Rowan-Robinson, M. 1985, ApJ, 298, L7

Herranz, D., Gallegos, J., Sanz, J. L., \& Martínez-González, E. 2002, MNRAS, 334, 533

Holder, G. P. 2002, ApJ, 580, 36

Ikebe, Y., Reiprich, T. H., Böhringer, H., Tanaka, Y., \& Kitayama, T. 2002, A\&A, 383, 773

Ikeuchi, S. 1981, PASJ, 33, 211

Jackson, C. A., \& Wall, J. V. 2001, in Particles and Fields in Radio Galaxies, ed. R. A. Laing, \& K. M. Blundell, ASP Conf. Proc., 250,400

Jackson, C. A., Wall, J. V., Shaver, P. A., et al. 2002, A\&A, 386, 97

Komatsu, E., \& Kitayama, T. 1999, ApJ, 526, L1

Kormendy, J., \& Gebhardt, K. 2001, in Proc. 20th Texas Symposium on relativistic astrophysics, ed. J. C. Wheeler, \& H. Martel, AIP Conf. Proc., 586, 363

Kovac, J. M., Leitch, E. M., Pryke, C., et al. 2002, Nature, 420, 772

Kühr, H., Witzel, A., Pauliny-Toth, I. I. K., \& Nauber, U. 1981, A\&AS, 45, 367

Lapi, A., Cavaliere, A., \& De Zotti, G. 2003, ApJ, 597, L93

Martínez-González, E., Diego, J. M., Vielva, P., \& Silk, J. 2003, MNRAS, 345, 1101

Mason, B. S., Pearson, T. J., Readhead, A. C. S., et al. 2003, ApJ, 591, 540

Massardi, M., \& De Zotti, G. 2004, A\&A, 424, 409

Mészáros, P. 1999, A\&AS, 138, 533

Morganti, R., Garrett, M. A., Chapman, S., et al. 2004, A\&A, 424, 371

Natarajan, P., \& Sigurdsson, S. 1999, MNRAS, 302, 288

Natarajan, P., Sigurdsson, S., \& Silk, J. 1998, MNRAS, 298, 577

O'Dea, C. P. 1998, PASP, 110, 493

Oh, S. P. 1999, ApJ, 527, 16

Pauliny-Toth, I. I. K., Witzel, A., Preuss, E., et al. 1978a, AJ, 83, 451

Pauliny-Toth, I. I. K., Witzel, A., Preuss, E., Baldwin, J. E., \& Hills, R. E. 1978b, A\&AS, 34, 253

Peacock, J. A. 1985, MNRAS, 217, 601

Peebles, P. J. E. 1993, Principles of physical cosmology (Princeton University Press)

Perna, R., \& Di Matteo, T. 2000, ApJ, 542, 68
Pierpaoli, E., Scott, D., \& White, M. 2001, MNRAS, 325, 77

Pierpaoli, E., Borgani, S., Scott, D., \& White, M. 2003, MNRAS, 342, 163

Platania, P., Burigana, C., De Zotti, G., Lazzaro, E., \& Bersanelli, M. 2002, MNRAS, 337, 242

Polatidis, A., Wilkinson, P. N., Xu, W., et al. 1999, NewAR, 43, 657

Press, W. H., Teukolsky, S. A., Vetterling, W. T., \& Flannery, B. P. 1992, Numerical recipes in FORTRAN. The art of scientific computing (Cambridge University Press)

Readhead, A. C. S., Taylor, G. B., Pearson, T. J., \& Wilkinson, P. N. 1996, ApJ, 460, 634

Rees, M. J., \& Ostriker, J. P. 1977, MNRAS, 179, 541

Rees, M. J., Phinney, E. S., Begelman, M. C., \& Blandford, R. D. 1982, Nature, 295, 17

Refregier, A., Spergel, D. N., \& Herbig, T. 2000, ApJ, 531, 31

Ricci, R., Sadler, E. M., Ekers, R. D., et al. 2004, MNRAS, submitted

Rosa-González, D., Terlevich, R., Terlevich, E., Friaça, A., \& Gaztañaga, E. 2004, MNRAS, 348, 669

Sadler, E. M., Jackson, C. A., Cannon, R. D., et al. 2002, MNRAS, 329, 227

Seaton, D. B., \& Partridge, R. B. 2001, PASP, 113, 6

Sheth, R. K., Mo, H. J., \& Tormen, G. 2001, MNRAS, 323, 1

Silva, L., De Zotti, G., Granato, G. L., Maiolino, R., \& Danese, L. 2004, A\&A, submitted [arXiv: astro-ph/0403166]

Silva, L., Granato, G. L., Bressan, A., \& Danese, L. 1998, ApJ, 509, 103

Snellen, I. A. G., Schilizzi, R. T., Miley, G. K., et al. 2000, MNRAS, 319,445

Sunyaev, R. A., \& Zeldovich, Y. B. 1972, Comm. Ap. Sp. Phys., 4, 173

Taylor, A. C., Grainge, K., Jones, M. E., et al. 2001, MNRAS, 327, L1

Taylor, G. B., Marr, J. M., Pearson, T. J., \& Readhead, A. C. S. 2000, ApJ, 541, 112

Tinti, S., Dallacasa, D., De Zotti, G., Celotti, A., \& Stanghellini, C. 2004, A\&A, accepted

Toffolatti, L., Argueso Gomez, F., De Zotti, G., et al. 1998, MNRAS, 297, 117

Toffolatti, L., Franceschini, A., Danese, L., \& De Zotti, G. 1987, A\&A, 184, 7

Trushkin, S. A. 2003, Bull. Spec. Astrophys. Obs. N. Caucasus, 55, 90 [arXiv: astro-ph/0307205]

Tschager, W., Schilizzi, R. T., Röttgering, H. J. A., Snellen, I. A. G., \& Miley, G. K. 2000, A\&A, 360, 887

Vielva, P., Martínez-González, E., Gallegos, J. E., Toffolatti, L., \& Sanz, J. L. 2003, MNRAS, 344, 89

Waldram, E. M., Pooley, G. G., Grainge, K. J. B., et al. 2003, MNRAS, 342, 915

Waxman, E. 1997, ApJ, 489, L33

White, S. D. M., \& Rees, M. J. 1978, MNRAS, 183, 341

Wijers, R. A. M. J., \& Galama, T. J. 1999, ApJ, 523, 177 